\title{
Incidental findings in lumbar spine MRI: their prevalence and potential impact on patient management
}

\author{
Heba Ibrahim * and Momena Essam Ibrahim Elsadawy
}

\begin{abstract}
Background: To document the prevalence of extra spinal findings in lumbar MRI

Results: Among the scanned 400 patients, 90 cases had incidental non-spinal findings, and in 30 out of these 90 patients, the finding was the only reason for their complaint.

Conclusions: Radiologists should give attention to the non-spinal findings in lumbar spine MRI, as the detected pathology could be the source of pain or could potential life-threatening conditions.
\end{abstract}

Keywords: Lumbar, MRI, Back pain, Spinal

\section{Background}

Low back pain is one of the most common complaints among a large variety of population [1]. The prevalence of chronic lower back pain is about $23 \%$, and the lifetime prevalence of lower back pain is estimated to be around $60-70 \%$ [2].

The majority of low back pain presentations represent musculoskeletal causes. Yet, some patients will present with other pathologies that can cause neurologic disability (i.e., abdominal or retroperitoneal etiologies).

In recent years, advances in digital evaluation of radiological imaging (e.g., high magnification zoom, the ability to focus on individual images, and digital archiving) have dramatically improved the detection limit of incidental lesions [3].

There are some studies that reviewed the frequency of incidental extra spinal findings in lumbar MRI and their clinical significance. Therefore, careful observation of anatomic structure outside of the immediate region of interest creates opportunities for early detection of potentially life-threatening conditions such as malignancies and aneurysms [4].

Non-spine-related causes of back pain may include vascular cases (abdominal aortic aneurysm (AAA), aortic

\footnotetext{
* Correspondence: hebaali_m@hotmail.com

Radio-diagnosis Department, Faculty of Medicine, Ain Shams University, Ramsis, Cairo, Egypt
}

\section{Methods}

Patients

The study was retrospective and approved by the local ethics committee. Due to the nature of the study design, there was no need to obtain specific informed consent from patients.

Four hundred patients were referred for lumbar spine MRI from the clinic of spine surgery, neurology, or orthopedic surgery. All had low back pain.

The patients aged 21 to 75 years, with a mean of $46 \pm$ 3 years. Two hundred-seventy of them were females and 130 were males (Fig. 1).

We excluded the patients who had spine pathology, known malignancy, or previous surgery and also any incomplete or low-quality scan. 


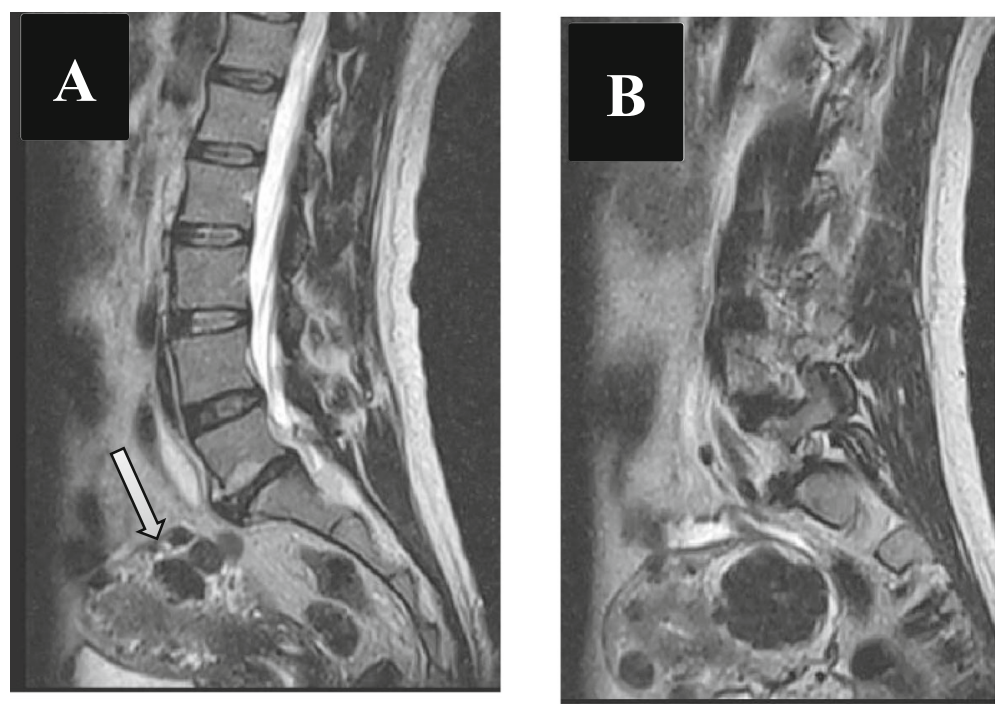

Fig. 1 47-year-old female with low back pain. Sagittal T2WI of the lumbar spine showing multiple interstitial uterine myomas seen in a (arrow). b shows large posterior wall myoma

The clinician examined the patients for neurological findings but all were referred with a symptom of back pain without any specific sign written in the radiology referral sheet.

Ten patients had pain referred to the hip joints, so complementary coverage of the hips was requested.

Thirteen patients were recommended to have a contrast study; only seven came back and received a contrast.

\section{Diagnostic workup and MR protocol}

The examination was conducted using $0.32 \mathrm{~T}$ system, Siemens Magnetom C, Germany, using a surface coil, and scanning was done in supine position with slight hip flexion with a pillow under the knee.

The scan included routine MR imaging pulse sequences: sagittal T2-weighted (3190/128/3) [TR/TE/number of excitation] and sagittal T1-weighted (790/17/3) turbo spinecho sequences, axial T1- and T2-weighted, coronal, and axial gradient 3D thin cuts. The slice thickness is $4 \mathrm{~mm}$ with an intersection gap of $1.4 \mathrm{~mm}$.

After the routine MR imaging, short scanning of the hip joints was conducted in 10 patients as per clinician request including sagittal T2 and coronal TIRM (T2 fat suppression).

Based on the radiologist's recommendation, 13 patients were called back for contrast study, seven of them came back and received contrast, and post-contrast T1WI was acquired in axial, sagittal, and coronal planes plus DIXON sequence (T1 with fat suppression).

Two patients were recommended to do MR spectroscopy for suspected prostate lesion; they referred to high field magnet to be done.

\section{Images interpretation}

After regular interpretation of the lumbar spine MRI using our facility PACS Hinai ImageNET, special concern was given to the extra spinal findings following a dedicated survey list including the presence of any extra spinal finding, the organ involved, the importance of such finding, the reliability of the lumbar MRI to diagnose that pathology, and finally how important and urgent was that finding to report to the referring physician.

Images were interpreted independently by two radiologists, 15 and 14 years of experience as general radiologist, guided by the tailored survey list. Data are collected and discussed with the referring physician for clinical correlation and further workup.

Six months and 1 year of follow up was done either by contacting the patient, patients coming for rescanning, or through the referring physician.

\section{Results}

We retrospectively included 400 patients with low back pain. Our exclusion criteria included patients with no extra spinal findings, patients with spine pathology, known malignancy or previous surgery, and also any incomplete or low-quality scan.

Ninety out of 400 patients (22.5\%) had extra spinal finding including 74 females (82\%) and $16(18 \%)$ males. Their ages ranged between 21 and 75 years, with a mean of $46 \pm 3$ years.

All patients presented with low back pain as per the referring physician with no other specific sign or symptoms. No previous investigations were done. 
Two radiologists interpreted the images and there was an agreement about detection and localization of the lesion.

Both radiologists could detect the extra spinal finding in the 90 included patients (100\%); they agreed about the organ of origin, except in suspected cases of metastases (10 cases), accuracy of localization $88.8 \%$, and in two cases of suspected metastases, they detected prostatic mass so they requested dedicated prostatic MRI with spectroscopy.

Regarding the organ involved, 40 of the 90 patients (44.5\%) had ovarian findings including simple or complex cyst and dermoid cyst or ovarian mass. Eighteen out of 90 patients (20\%) had uterine myomas (Fig. 2). Fifteen out of 90 patients $(16.5 \%)$ had renal cysts. Two out of 90 patients $(2.3 \%)$ had paraspinal masses like the lymph node, ten out of 90 (11\%) had lumbar and sacral metastases, seven out of 90 (7.7\%) had hip lesions (avascular necrosis (AVN) and insufficiency fracture), and three out of 90 had aortic aneurysm. Two of the cases showed paraspinal masses (2.2\%), one of them revealed to be psoas abscess (Pott's disease), and the other one revealed to be neurofibroma.

There was concordance about the suspected diagnosis (Table 1). The requested further investigation was either to complete the diagnosis (in twenty-one out of 90 cases, 23\%) or to better assess the size and relationship as in cases of ovarian and uterine lesions where pelvic ultrasound was requested for all of them for more information.

Twenty-one out of 90 patients (23\%) needed further contrast study, 20 patients (22\%) needed MRI with contrast ( 5 cases of complex ovarian cyst, 3 cases of ovarian masses, 2 cases of paraspinal masses, and 2 cases of metastases), and one case needed CT angiography for abdominal aortic aneurysm.

Ten patients (11\%) submitted to metastatic work up to search for the primary and for staging.

All cases of ovarian or uterine lesions were recommended to do pelvic ultrasound, not for diagnosis but
Table 1 Showing the detected extra spinal findings

\begin{tabular}{ll}
\hline Number of findings & Extra spinal findings \\
\hline 18 & Simple ovarian cyst \\
7 & Complex \\
12 & Dermoid cyst \\
3 & Ovarian mass \\
18 & Uterine myoma \\
15 & Renal cyst \\
2 & Paraspinal mass \\
10 & Metastases \\
7 & Hip lesions \\
3 & Aortic aneurysm \\
\hline
\end{tabular}

for more information about the lesion including its size and relationship.

In 10 out of 90 patients (11\%), the extra spinal finding was the main cause of low back pain, and symptoms subsided after surgical intervention. Three of them were posterior wall subserous myoma, four of them were ovarian lesions (Fig. 2), and three out of 90 were paraspinal masses.

Regarding the lumbar spine MRI alone, it was enough and reliable in the diagnosis of the detected lesion in sixty-nine out of 90 (75\%) patients, the performed MRI was enough to give a final diagnosis, and no further imaging was needed to diagnose the pathology. However, the recommended imaging (pelvic ultrasound) was to better overall assess the condition regarding the size and extension of the lesion.

Regarding the urgency of reporting, six out of 90 patients $(6.6 \%)$ were urgently reported to the referring clinician within $12 \mathrm{~h}$ including one case of huge aortic aneurysm, two cases of paraspinal masses, and three cases of hip lesions (Fig. 3) (insufficiency fracture and AVN).

\section{Discussion}

Low back pain is one of the most common reasons for an outpatient visit. The evaluation for low back pain
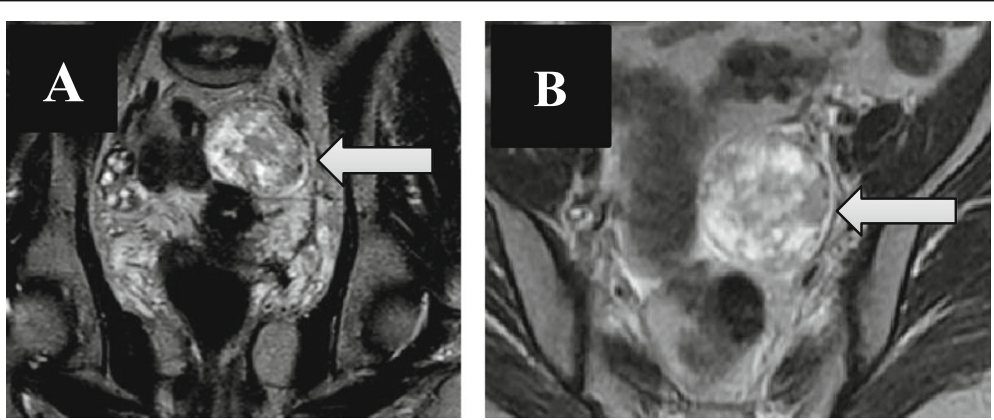

Fig. 2 39-year-old female patient with low back pain. a Coronal T2WI showing left adnexal heterogenous mass. b Axial T2WI shows the mass at the left ovary. Histopathology revealed left ovarian thecoma 

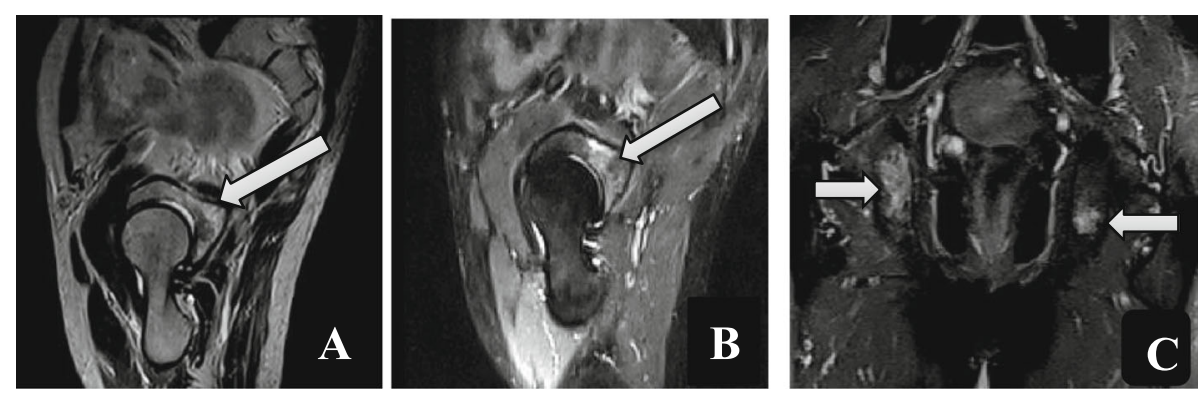

Fig. 3 62-year-old male patient with back pain radiating to the hip joints. a is sagittal T2WI of the left hip joint showing bone marrow edema of the acetabulum (arrow), $\mathbf{b}$ is TIRM confirming the acetabular edema, and $\mathbf{c}$ is coronal T2 TIRM of both hip joints showing bilateral acetabular edema consistent with insufficiency fracture

should include a complete, focused medical history [5]. Incidental imaging findings in lumbar MRI are common and may lead to further tests or investigations [6].

General radiologists frequently report lumbar MRI and they should be aware and familiar with any extra spinal abnormalities either for their clinical significance, medico legality, or for improving our practice as these findings are not uncommon. So, it is generally recommended to have a systematic reporting approach to avoid missing any finding.

We aimed to highlight some of the detected extra spinal pathologies to avoid tunnel view to lumbar MRI; we assessed the prevalence, organ of origin, suspected diagnosis, and correlation with patient symptomatology and level of threat of the detected abnormalities.

In our study, the extra spinal findings were detected in $22.5 \%$ of cases submitted to lumbar MRI for low back pain. Sedat et al. [7] reported a prevalence of $19.8 \%$ incidental extra spinal findings in routine lumbar MRI, and they stated that their rate is less than the values reported in the literature. Lee et al. [4] reported a rate of $40.5 \%$ of incidental extra spinal findings in lumbar MRI. This variability may be attributed to technical factors, like FOV, coverage, and using PACS for interpretation.

Our study included more females than males (82\%), in agreement with the literature. The majority (44.5\%) of our findings were ovarian in origin followed by uterine pathologies, in comparison to Sedat et al. [7] where most of the findings are renal in origin, and they focused more about size criteria of the detected renal cyst. Forty-one percent of our patients had ovarian cysts, either simple, complicated, or dermoid cysts. The simple cysts represent most of the ovarian lesions; they displayed homogenous hypointensity on T1WI and hyperintensity on T2WI with no wall thickening. Renal cysts account for $17 \%$ of our findings.

Complex cysts are mostly hemorrhagic; they are bright on T1WI indicating either fat or blood content, on sat suppressed T1WI; and they remain bright, ruling out a fatty lesion. If the contrast is given, there will be no enhancement [5].

Twenty percent of cases had uterine myomas, being the most common are benign gynecologic tumors. They primarily affect women of reproductive age, and the estimated incidence of fibroids is over $70 \%$ by 50 years of age [7]. Although submucosal leiomyomas are the least common, they are most commonly symptomatic, and also, pedunculated subserous leiomyomas may undergo torsion, which results in infarction accompanied by pain [8].

We had 10 cases $(10 \%)$ of osseous metastasis. Indeed, bone is the third most common organ affected by metastasis, surpassed only by the lungs and liver [9]. Approximately $70 \%$ of patients with breast or prostate cancer have bone metastases [10].

$\mathrm{Ju} \mathrm{Fu}$ et al. [11] focused on the incidental extra spinal malignancies found on lumbar MRI, the majority of their cases were lymph nodes, they reported $32.1 \%$ of their study population as lymphadenopathy either metastases or lymphoma, and our study included only three cases of lymph nodes suspected of metastases (3\% of the total population).

Three out of 90 cases (3\%) showed an abdominal aortic aneurysm. Abdominal aortic aneurysms occur most commonly in individuals between 65 and 75 years of age. They often do not cause any symptoms and are found incidentally on physical examination or imaging examinations of the abdomen and pelvis [12]. Approximately $90 \%$ of all abdominal aortic aneurysms are infrarenal [13].

Seven out of 90 cases (8\%) found to have hip lesions, namely femoral head avascular necrosis and acetabular fracture. The incidence of AVN has been increasing. The causes include greater use of exogenous steroids and an increase in trauma [14]. MRI signs include subchondral edema, low signal serpiginous line, and double line sign (inner bright line from granulation tissue and outer dark line from sclerotic bone) on T2-weighted images $[15,16]$. 
Clinician/radiologist feedback was very crucial as the radiological opinion guided the clinician to further assessment and the radiologists got a short and long feedback from the clinician following the patient.

We had some limitations including the inability to do all requested examinations in the same place, and the study being retrospective did not facilitate on time complementary ultrasound correlation.

We found that MRI of the lumbar spine could convey a lot of information apart from just spine imaging, and lumbar MRI alone could be reliable in some extra spinal pathologies, could raise doubt in some other, and even warn against life-threatening or serious pathologies.

\section{Conclusion}

Extraspinal incidental findings are not uncommon and sometimes may explain patient symptomatology.

The main recommendation of this study is to avoid the tunnel vision interpretation of the lumbar spine and to draw the radiologist's attention towards other scanned non-spinal organs during reading lumbar spine MRI, either this incidental finding was significant or not. In addition, encouraging clinician-radiologist feedback, discussion, and further workup of certain cases are needed.

\section{Abbreviations}

MRI: Magnetic resonance imaging

\section{Acknowledgements}

The authors thank all radiographers for their support.

\section{Authors' contributions}

IH suggests the research idea, reviewing the literature, and data collection and analysis; perform statistical analysis; revise the manuscript; and prepared the MRI cases. EM contributed to the reviewing of the literature and wrote the manuscript and final editing. All authors have read and approved the final manuscript.

\section{Funding}

This study had no funding from any resource.

\section{Availability of data and materials}

The datasets used and/or analyzed during the current study are available from the corresponding author on reasonable request.

\section{Ethics approval and consent to participate}

This study was approved by the local Research Ethics Committee. It was a retrospective study, and no consent was taken from the patients.

\section{Consent for publication}

Not applicable

\section{Competing interests}

The authors declare that they have no competing interests.

Received: 11 July 2019 Accepted: 26 September 2019 Published online: 26 November 2019

\section{References}

1. Friedman BW et al (2010) Diagnostic testing and treatment of low back pain in US Emergency Departments. A National Perspective. Spine 35(24): E1406-E1411

2. Balague $F$ et al (2011) Non-specific low back pain. The Lancet 379(9814): 482-491. 21982256
3. Wagner SC, Morrison WB, Carrino JA, Schweitzer ME, Nothnagel H (2002) Picture archiving and communication system: effect on reporting of incidental findings. Radiology. 225:500-505

4. Lee SY, Landis MS, Ross IG, Goela A, Leung AE (2012) Extraspinal findings at lumbar spine CT examinations: prevalence and clinical importance. Radiology. 263:502-509

5. Bigos S, Bowyer O, Braen G et al (2012) Acute low back problems in adults. Radiology 95:0642

6. Levine D et al (2010) Management of asymptomatic ovarian and other adnexal cysts imaged at US: society of radiologists in ultrasound consensus conference statement. Radiology 256:943-954

7. Sedat Alpaslan Tuncel MD, Bekir Çaglı MD, Aslan Tekataş MD, Mehmet Yadigar Kırıcı MD, Ercüment Ünlü MD, Hakan Gençhellaç MD (2015) Extraspinal incidental findings on routine MRI of lumbar spine: prevalence and reporting rates in 1278 patients. Koran J of Radiology. 16(4):866-873

8. Spencer JA1, Forstner R, Cunha TM, Kinkel K (2010) ESUR guidelines for MR imaging of the sonographically indeterminate adnexal mass: an algorithmic approach. Eur Radiol. 20(1):25-35

9. Sue W, Sarah S-B (2009) Radiological appearances of uterine fibroids. Indian J Radiol Imaging. 19(3):222-231

10. Elizabeth Kagan Arleo1, Peter E. Schwartz2, Pei Hui3 and Shirley McCarthy4 (2017) Uterine leiomyoma (Fibroid) imaging, Updated;34-45.

11. Chen-Ju F, Chen H-W, Wu C-T, Chen L-H, Wong Y-C, Wang L-J, Wan Y-L, YiKang K (2013) Extraspinal malignancies found incidentally on lumbar spine MRI: prevalence and etiologies. J Radiol Sci 38:85-91

12. Cuccurullo V, Cascini GL, Tamburrini O, Rotondo A, Mansi L (2013) Bone metastases radiopharmaceuticals: an overview. Curr Radiopharm. 6:41-47

13. Choi J, Raghavan M (2012) Diagnostic imaging and image-guided therapy of skeletal metastases. Cancer Control. 19:102-112

14. Keisler B, Carter C (2015) Abdominal aortic aneurysm. Am Fam Physician. 91(8):538-543

15. Ajay D. Wadgaonkar, MD James H. Black III, MD Elizabeth K. Weihe, MD Stefan L. Zimmerman, MD Elliot K. Fishman, MD Pamela T. Johnson, MD. Abdominal aortic aneurysms revisited: MDCT with multiplanar reconstructions for identifying indicators of instability in the pre- and postoperative patient, Volume 35, Issue 1, January, 2015; 680-700.

16. Michael R Aiello, MD and Felix S Chew, MD. (2017) Isssmaging in avascular necrosis of the femoral head, 11, i20-128.

\section{Publisher's Note}

Springer Nature remains neutral with regard to jurisdictional claims in published maps and institutional affiliations.

\section{Submit your manuscript to a SpringerOpen ${ }^{\circ}$ journal and benefit from:}

- Convenient online submission

- Rigorous peer review

- Open access: articles freely available online

High visibility within the field

- Retaining the copyright to your article

Submit your next manuscript at $>$ springeropen.com 